D. Briggs sscL.512 new sw 9409

Low Frequency Transverse Resistive Instability in the Collider

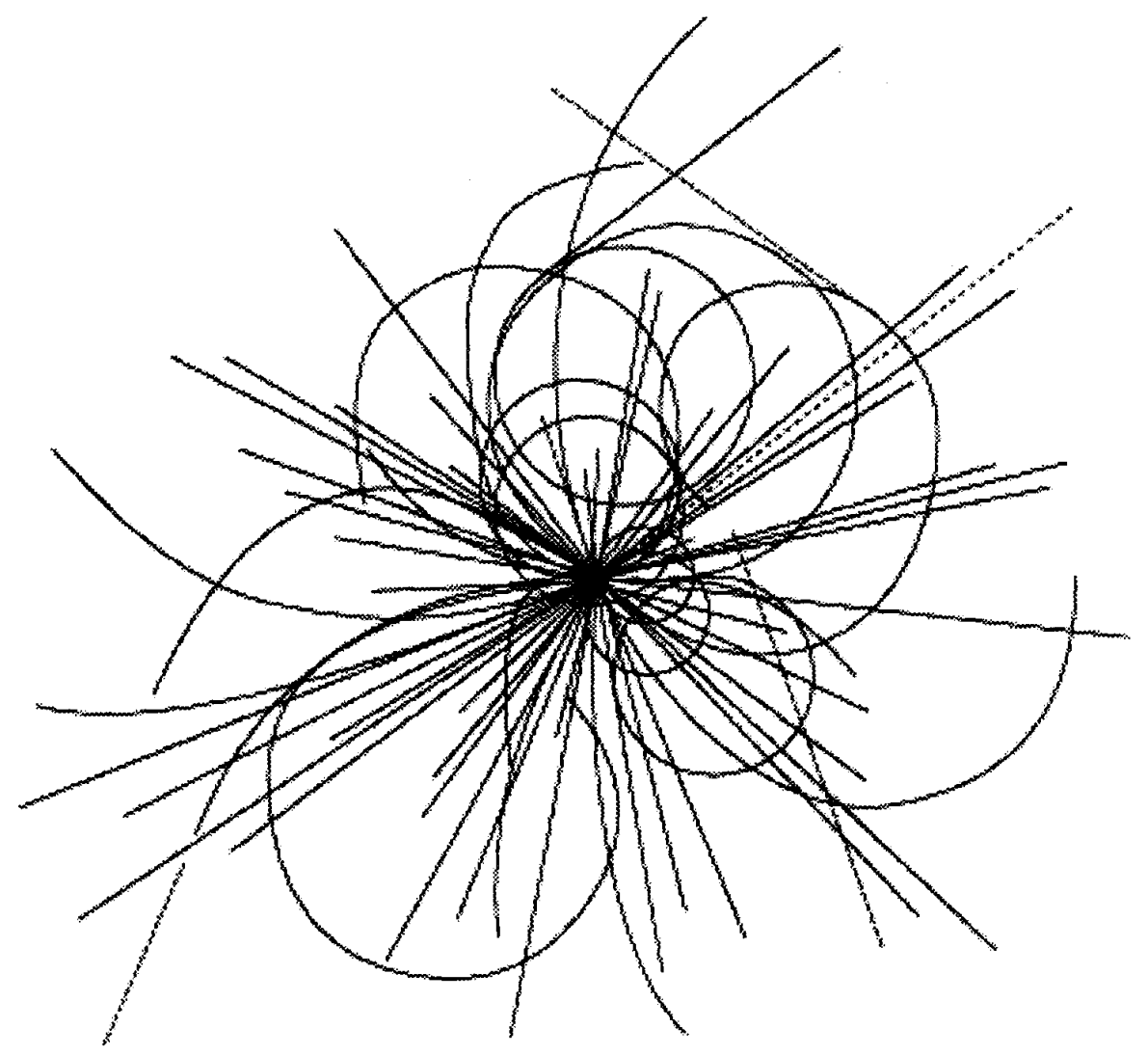

Superconducting Super Collider Laboratory 


\section{Disclaimer Notice}

This report was prepared as an account of work sponsored by an agency of the United States Govemment. Neither the United States Government or any agency thereof, nor any of their employees, makes any warranty, express or implied, or assumes any legal liability or responsibility for the accuracy, completeness, or usefulness of any information, apparatus, product, or process disclosed, or represents that its use would not infringe privately owned rights. Reference herein to any specific commercial product, process, or service by trade name, tradernark, manufacturer, or otherwise, does not necessarily constitute or imply its endorsement, recommendation, or favoring by the United States Government or any agency thereof. The views and opinions of authors expressed herein do not necessarily state or reflect those of the United States Government or any agency thereof.

Superconducting Super Collider Laboratory is an equal opportunity employer. 
SSCL-512 Rev. 1

\title{
Low Frequency Transverse Resistive Instability in the Collider
}

\author{
D. Briggs \\ Superconducting Super Collider Laboratory* \\ 2550 Beckleymeade Ave. \\ Dallas, TX 75237 USA \\ June 1992
}

*Operated by the Universities Research Association, Inc., for the U.S. Department of Energy under Contract No. DE-AC35-89ER40486. 



\subsection{INTRODUCTION}

The large scale size of the Superconducting Super Collider (SSC) collider rings makes it possible for the multibunch resistive instability to occur at very low frequencies $(e . g$., less than $1 \mathrm{kHz})$. For the lowest allowed frequencies, the skin depth is generally larger than the thickness of the copper layers that will be placed on the inner diameter of the beam tube, or on a synchrotron radiation intercept tube (liner) if a decision is made to go that route. The regime of skin depth larger than the thickness of the conducting beam tube was considered earlier by J. D. Jackson (Ref. 1). Jackson allowed for two concentric conducting layers on the tube, but he assumed that the fields vanish outside the pipe. In this note, we generalize Jackson's results to arbitrary values of the conductivity, to show the dependence of the interaction impedance on copper layer thickness, or frequency, over the full range. The main purpose of this note is to present a general formulation of the interaction impedance that connects the various limiting cases. In particular, this formulation predicts a maximum value for the instability growth rate ( $R e$ $Z_{\perp}$ ) as a function of thickness, or frequency.

In Section 2.0, a simplified derivation of the multibunch transverse instability growth rate in the low frequency regime is given, for completeness. Conditions for stabilization with a spread in the betatron tune are also derived. In Section 3.0, the general expression for low-frequency interaction impedance is developed. In Section 4.0, we discuss the requirements on copper layer thickness to limit the growth rate of the mode to acceptable values in the SSC collider rings. The conditions for stabilization of the mode with space-charge tune spread at injection, or beam-beam tune spread at full energy, are also given.

\subsection{GROWTH RATE IN TERMS OF $\mathrm{Z}_{\perp}$}

The most dangerous regime for the multibunch transverse resistive instability is usually the lowest (allowed) frequencies, where the fields have the greatest diffusion through the conducting pipe (or liner). In this regime, the actual beam structure consisting of discrete bunches spaced $\Delta z \sim 5 \mathrm{~m}$ apart can be treated as a continuum. The dipole moment due to transverse particle displacements at time $t$ and axial coordinate $z$ (in the direction of beam motion) is proportional to $I\left(t-\frac{z}{c}\right) Y_{b}(t, z)$, where $I$ is the instantaneous current and $Y_{b}$ is the coherent transverse beam displacement. If we expand $I$ in a Fourier series, we see that the slowly-varying EM fields responsible for the resistive growth are proportional to $I_{b} Y_{b}$, where

$$
I_{b}=\langle I\rangle
$$

is the average beam current. For a given beam environment, we characterize the transverse coherent force by the transverse interaction impedance $Z_{\perp}$, defined by ${ }^{1}$

$$
\begin{aligned}
\bar{F}_{\perp}(z, t) & =e(\bar{E}+\bar{V} \times \bar{B})_{\perp} \\
& =-\frac{j e I_{b} Y_{b}}{2 \pi R} Z_{\perp} .
\end{aligned}
$$

Here we take the dependence $\exp j(\omega t-k z)$ for the displacement $\left(Y_{b}\right)$ and the fields, and define $2 \pi R$ as the ring circumference. (We use electrical engineering notation for the complex amplitudes, preferred to keep impedance definitions in their traditional form.)

If we describe the transverse focusing system by an average beta function $\left(\beta_{\text {ave }}\right)$, the coherent transverse motion of the beam particles is governed by the force equation: 


$$
\frac{1}{c^{2}} \frac{d^{2} Y_{b}}{d t^{2}}+k_{\beta}^{2} Y_{b}=\frac{F_{\perp}}{m_{m_{o}} c^{2}}
$$

Here, $k_{\beta}=\left(\beta_{a v e}\right)^{-1}, \frac{d}{d t}=\frac{\partial}{\partial t}+\mathrm{v} \frac{\partial}{\partial z}, \mathrm{c}$ is the speed of light $(\mathrm{v} \approx \mathrm{c})$, and $\gamma m_{o} c^{2}$ is the proton energy. With the assumed $z, t$ dependence, we have the following dispersion equation, obtained by substituting Eq. (1) into Eq. (2):

$$
\left(\frac{\omega}{c}-k-k_{\beta}\right)\left(\frac{\omega}{c}-k+k_{\beta}\right)=\frac{j e Z_{\perp} I_{b}}{2 \pi R m_{o} c^{2}}
$$

The unstable root has $\frac{\omega}{c} \approx k-k_{\beta}$, and the approximate solution for $\omega$ assuming the coherent forces are small (i.e., assuming we don't have disastrous growth rates) is

$$
\omega=\left(k-k_{\beta}\right) c-\frac{j e Z_{\perp} I_{b}}{4 \pi R m_{o} c k_{\beta}} .
$$

Since all variables satisfy the periodicity condition $A(z+2 \pi R)=A(z)$, the axial wavenumber is quantized as $k(2 \pi R)=2 \pi n$. The betatron tune is defined by $k_{\beta}(2 \pi R)=2 \pi v$. Introducing the particle revolution frequency $\omega_{o}=c / R$, we can write the following expression for $\omega / \omega_{o}$ :

$$
\frac{\omega}{\omega_{o}}=n-v-j \frac{I_{b}}{I_{o}} \frac{Z_{\perp}}{Z_{o}} \frac{\beta_{\text {ave }}}{\gamma} .
$$

Here we define $I_{o}=4 \pi m_{o} c / \mu_{o} e, Z_{o}=\left(\mu_{o} \varepsilon_{o}\right)^{1 / 2}$, and we replace $1 / \mathrm{k}_{\beta}$ by $\beta_{\text {ave. }}$ The (real) frequency of the mode is given by the usual expression,

$$
\operatorname{Re} \omega=(n-v) \omega_{o}
$$

while the growth rate, $\omega_{g}=-\operatorname{Im} \omega$, is

$$
\frac{\omega_{g}}{\omega_{o}}=\frac{I_{b}}{I_{o}} \frac{\beta_{a v e}}{\gamma Z_{o}} \operatorname{Re}\left(Z_{\perp}\right)
$$

The expression given here for the growth rate agrees with that given by Eq. (4.5-25) in the "Blue Book" (Ref. 2). To make the connection, note that the average current is $I_{b}=M N_{B} e / \tau_{o}$, where $\tau_{o}=2 \pi / \omega_{o}$ is the revolution period, and the classical radius of the proton is $r_{p}=e c / I_{o}=e^{2} \mu_{o} / 4 \pi m_{o}$.

A spread in betatron tunes can stabilize the resistive instability. To develop a criterion for stabilization, we use a "multibeam" model (Ref. 3) where $Y_{\mathrm{bi}}$ is the "beamlet" with focusing strength $k_{\beta i}$ or tune $v_{\mathrm{i}}=$ $k_{\beta i} R$. The fraction of particles with tune $v_{\mathrm{i}}$ is given by $f\left(v_{\mathrm{i}}\right) d v_{\mathrm{i}}$, where

$$
\int f\left(v_{i}\right) d v_{i}=1 .
$$


Each beamlet satisfies the force equation, (Eq. 2), using the net EM fields obtained by summing over the dipole contributions of all the beamlets, namely

$$
F_{\perp}=\frac{-j e I_{b} Z_{\perp}}{2 \pi R} \int Y_{b i} f\left(v_{i}\right) d v_{i}
$$

Now, from the force equation,

$$
-\left(\frac{\omega}{c}-k-k_{\beta i}\right)\left(\frac{\omega}{c}-k+k_{\beta i}\right) Y_{b i}=\frac{F_{\perp}}{m_{o} c^{2}}
$$

we obtain the dispersion equation

$$
1=-j \frac{I_{b} Z_{\perp} \beta_{a v e}}{I_{o} Z_{o} \gamma} \int \frac{f\left(v_{i}\right) d v_{i}}{\frac{\omega}{\omega_{o}}-n+v_{i}}
$$

as the generalization of Eq. (5) to include a betatron frequency spread. In deriving Eq. (11) we have assumed that $\frac{\omega}{\omega}$ is close to $n-<v>$ for the (potentially) unstable modes.

The condition for marginal stability is obtained when $\omega$ is pure real; in this case, the integral over $v_{\mathrm{i}}$ leads to

$$
\int \frac{f\left(v_{i}\right) d v_{i}}{v_{i}-n+\frac{\omega}{\omega_{0}}}=j \pi f\left(n-\frac{\omega}{\omega_{0}}\right)+P \int \frac{f\left(v_{i}\right) d v_{i}}{v_{i}-n+\frac{\omega}{\omega_{0}}}
$$

where the second term on the right side is the principal part of the integral (a real term).

An important special case is purely resistive impedance, and a distribution that is symmetrical about a mean tune $v_{0}$ (see Figure 1). In this case, the marginal stability condition is satisfied by the real frequency $\frac{\omega}{\omega_{0}}=n-v_{0}$, which makes the principal part integration vanish by symmetry. Defining

$$
f\left(v_{o}\right)=\frac{1}{\Delta v_{\mathrm{s}}}
$$

where $\Delta v_{\mathrm{s}}$ is a measure of the width of the distribution (see Figure 1), the stabilization condition becomes (from Eqs. 11 and 12)

$$
\Delta v_{s} \geq \pi \frac{I_{b} Z_{\perp} \beta_{\text {ave }}}{I_{o} Z_{o} \gamma}
$$


In more general cases with complex $Z_{\perp}$, the betatron tune spread required for stabilizing the resistive mode is of the same order as Eq. (14), if we use the magnitude of $Z_{\perp}$ on the right hand side.

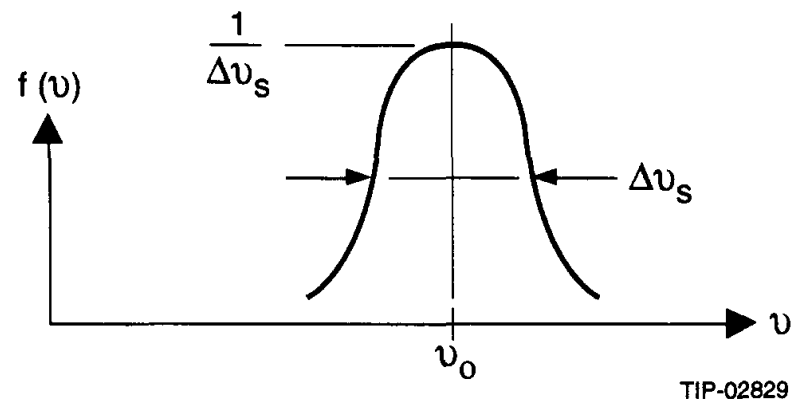

Figure 1. Betatron Tune Distribution.

\subsection{CALCULATION OF $\mathbf{Z}_{\perp}$}

The dipole fields created by a coherent oscillation of the beam in the $y$-direction are given by

$$
\begin{aligned}
& \overline{E_{b}}=\frac{Z_{o} I_{b} Y_{b}}{2 \pi r^{2}}\left(\sin \theta \overline{a_{r}}-\cos \theta \overline{a_{\theta}}\right) \\
& \overline{H_{b}}=\frac{I_{b} Y_{b}}{2 \pi r^{2}}\left(\cos \theta \overline{a_{r}}+\sin \theta \overline{a_{\theta}}\right)
\end{aligned}
$$

for low frequencies and long wavelengths, and $\mathrm{v} \approx \mathrm{c}$. To account for the presence of a conducting liner or wall at a radius $r=b$, we must add the fields created by wall currents and charges to the above in the $r<b$ region to satisfy the boundary conditions; namely

$$
\begin{aligned}
& \overline{E_{w}}=E_{w}\left(\sin \theta \overline{a_{r}}+\cos \theta \overline{a_{\theta}}\right)=E_{w} \overline{a_{y}} \\
& \overline{H_{w}}=H_{w}\left(\cos \theta \overline{a_{r}}-\sin \theta \overline{a_{\theta}}\right)=H_{w} \overline{a_{x}} .
\end{aligned}
$$

The region $r \geq b$ can be described by an effective "wall impedance,"

$$
Z_{w}=\left(\frac{-E_{z}}{H_{\theta}}\right)_{r=b}
$$

that we calculate below for several cases of interest.

The axial electric field is obtained from Maxwell's equations; for example, from

$$
\frac{1}{r} \frac{\partial E_{z}}{\partial \theta}-\frac{\partial E_{\theta}}{\partial z}=-j \omega \mu_{o} H_{r} .
$$


In Appendix I, the full solution for the transverse fields are obtained, to show that the contribution from the $E_{\theta}$ term in $E_{z}$ can be neglected, and that $E_{\theta}=0 a t r=b$ gives a good approximation to the transverse electric field in all cases of interest. The physical reason for this result is that the charge relaxation time scale $\left(\varepsilon_{o} b Z_{w}\right)$ is infinitesimal compared to the other timescales of interest $(1 / \omega)$ for metal layer thicknesses of practical significance.

From Eq. (20), neglecting $E_{\theta}$,we have

$$
E_{z}=-j \omega \mu_{o} \sin \theta\left[r H_{w}+\frac{I_{b} Y_{b}}{2 \pi r}\right] .
$$

From Eqs. (16),(18), (19) and (21), we can solve for $H_{w}$ in terms of $Z_{w}$ as

$$
H_{w}=\frac{I_{b} Y_{b}}{2 \pi b^{2}}\left[\frac{Z_{w}-j \omega \mu_{o} b}{Z_{w}+j \omega \mu_{o} b}\right] .
$$

Similarly, applying the boundary condition $E_{\theta}(r=b)=0$ yields

$$
E_{w}=\frac{Z_{o} I_{b} Y_{b}}{2 \pi b^{2}}
$$

The fields from the wall currents and charges are the ones that contribute to the collective transverse force, so

$$
F_{\perp y}=e\left(E_{w}+c \mu_{o} H_{w}\right)
$$

and from Eqs. (22) and (23),

$$
F_{\perp y}=\frac{e Z_{o} I_{b} Y_{b}}{\pi b^{2}}\left(\frac{Z_{w}}{Z_{w}+j \omega \mu_{o} b}\right)
$$

From the definition of transverse interaction impedance (Eq. 1) we have

$$
\frac{Z_{\perp}}{Z_{o}}=j \frac{2 R}{b^{2}}\left(\frac{Z_{w}}{Z_{w}+j \omega \mu_{o} b}\right) .
$$

(a) The usual situation considered in resistive instability calculations is a conducting wall at $r=b$ with a thickness $\Delta$ that is much larger than the skin depth,

$$
\delta=\left(\frac{2}{\omega \mu_{o} \sigma}\right)^{\frac{1}{2}}
$$

In this regime,

$$
Z_{w}=\frac{1+j}{\sigma \delta}
$$


and we note that

$$
\frac{\left|Z_{w}\right|}{\omega \mu_{o} b} \simeq \frac{\delta}{2 b}
$$

is much less than one. We then have

$$
Z_{\perp}=\frac{2 c R(1+j)}{\omega b^{3} \sigma \delta},
$$

which is the usual result.

(b) The lowest instability frequencies in SSC, $(n-v) \omega_{0} \sim(0.1-0.3) \omega_{o}$, correspond to skin depths that are larger than the thickness of the copper layer on the liner or beam tube. To cover this case, we consider a single cylinder of thickness $\Delta$ at $r=b$ that dominates the magnetic field diffusion. (This is typically the copper layer on the beam tube.) The magnetic field outside the cylinder is of the form

$$
\bar{H}=H_{o} \frac{b^{2}}{r^{2}}\left[\cos \theta \overline{a_{r}}+\sin \theta \overline{a_{\theta}}\right],
$$

while the transverse electric fields are negligible. The axial electric field follows from Eq. (20):

$$
E_{z}=-j \omega \mu_{o} H_{o} \frac{b^{2}}{r} \sin \theta .
$$

We assume $\delta>\Delta$, so the axial current in the cylinder is approximately uniform, and the thin cylinder is equivalent to a "sheet" current layer with $K_{z}=\sigma \Delta E_{z}$. The continuity conditions relating the outside fields to the inside fields are

$$
\begin{gathered}
E_{z}\left(b^{+}\right)=E_{z}\left(b^{-}\right) \\
H_{\theta}\left(b^{+}\right)-H_{\theta}\left(b^{-}\right)=\sigma \Delta E_{z}(b) .
\end{gathered}
$$

From Eqs. (31) and (32) we have

$$
H_{\theta}\left(b^{+}\right)=H_{o} \sin \theta=-\frac{E_{z}(b)}{j \omega \mu_{o} b},
$$

which, with Eq. (34), gives the surface impedance

$$
Z_{w}=\left[-\frac{E_{z}(b)}{H_{\theta}\left(b^{-}\right)}\right]=\frac{1}{\sigma \Delta+\frac{1}{j \omega \mu_{o} b}} .
$$


Using this result in Eq. (26), the interaction impedance in the present case is given by

$$
\frac{Z_{\perp}}{Z_{o}}=\frac{j R}{b^{2}\left(1+j \omega \tau_{m}\right)},
$$

where we define

$$
\tau_{\mathrm{m}}=\frac{1}{2} \mu_{o} \sigma \Delta b
$$

The time constant $\tau_{\mathrm{m}}$ can be recognized as the "L/R" time for dipole currents in a thin conducting tube. Note also that the magnetic field outside the tube is related to the inside field as

$$
\frac{H_{\theta}\left(b^{+}\right)}{H_{\theta}\left(b^{-}\right)}=\frac{1}{1+2 j \omega \tau_{m}}
$$

and this ratio is small when $\omega \tau_{\mathrm{m}} \gg 1$, even if the skin depth is large compared to the thickness $\Delta$. (When $\delta$ is less than $\Delta$, of course, the outside magnetic field is smaller than the inner field by an exponential factor.)

From Eq. (37), we have

$$
\frac{\operatorname{Re} Z_{\perp}}{Z_{o}}=\frac{R}{b^{2}} \frac{\omega \tau_{m}}{1+\left(\omega \tau_{m}\right)^{2}}
$$

The resistive instability growth rate is proportional to $R e Z_{\perp}$, and it follows from Eq. (40) that the maximum possible growth rate as a function of $\Delta$, or $\omega$, occurs at $\omega \tau_{\mathrm{m}} \approx 1$, where $\frac{\operatorname{Re} Z_{\perp}}{Z_{o}}=\frac{R}{2 b^{2}}$.

The general form of the real part of the interaction impedance versus $\omega$ can now be pieced together from these limiting cases. Note that the ratio $\Delta \delta$ is of the form

$$
\frac{\Delta}{\delta}=\left(\frac{\omega \mu_{o} \sigma \Delta^{2}}{2}\right)^{1 / 2} \equiv\left(\omega \tau_{\mathrm{s}}\right)^{1 / 2}
$$

where $\tau_{\mathrm{s}}=\mu_{o} \sigma \Delta^{2} / 2$ is the "magnetic skin time." In the regime $\omega \tau_{\mathrm{s}}>>1$, the expression for $Z_{\perp}$ (Eq. 30) can be put in the form

$$
\frac{\operatorname{Re} Z_{\perp}}{Z_{o}}=\frac{R}{b^{2}} \frac{\sqrt{\omega \tau_{s}}}{\omega \tau_{m}}
$$


In the region $\frac{1}{\tau_{\mathrm{m}}} \ll \omega \ll \frac{1}{\tau_{\mathrm{s}}}$, we have from Eq. (40):

$$
\frac{\operatorname{Re} Z_{\perp}}{Z_{o}}=\frac{R}{b^{2}} \frac{1}{\omega \tau_{\mathrm{m}}}
$$

A sketch of $\operatorname{Re} Z_{\perp}$ as a function of frequency is presented in Figure 2, and as a function of thickness ( $\Delta$ ) at a fixed frequency in Figure 3, to illustrate the connection between these limiting cases.

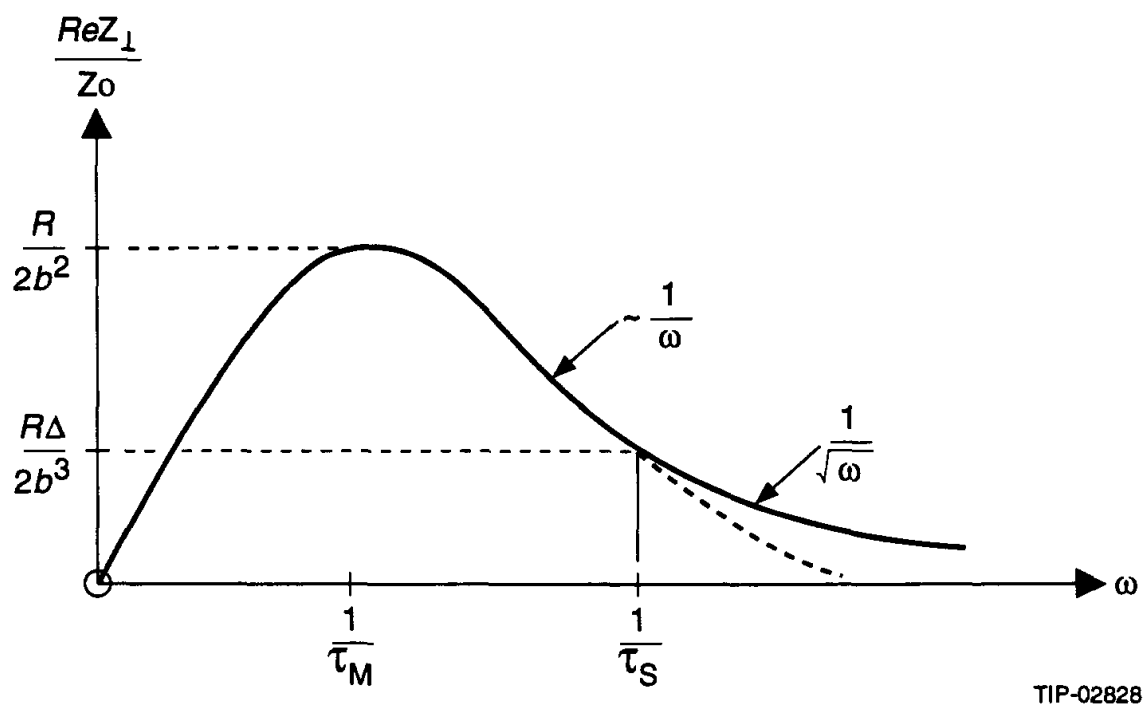

Figure 2. Interaction Impedance versus $\omega$.

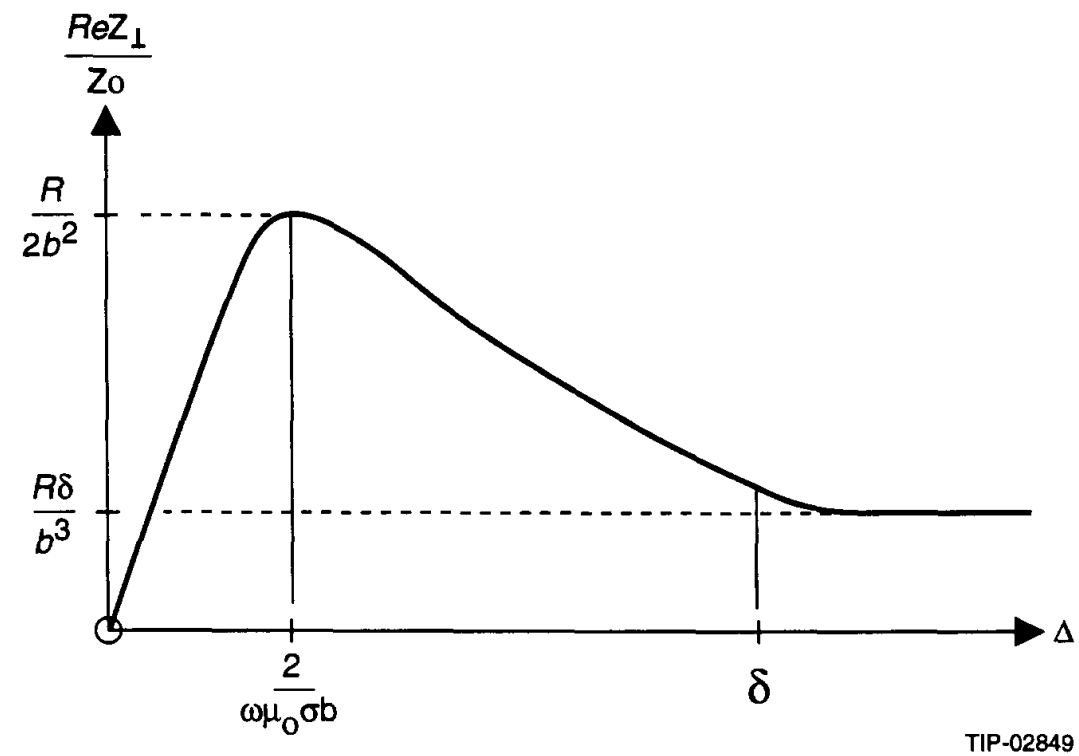

Figure 3. Interaction Impedance versus $\Delta$. 


\subsection{APPLICATION TO THE SSC COLLIDER}

As shown in Section 3.0, the resistive instability growth rate has a very broad maximum around $\omega \tau_{m}=1$. Reasonable choices for the thickness of the copper coating on the beam tube (or liner) can result in $\omega \tau_{m} \gg 1$ even for the lowest allowed frequencies; however, it is useful to first consider the maximum possible growth rate as a benchmark. Using the maximum $\operatorname{Re} Z_{\perp} / Z_{o}$ from Eq. (40) in Eq. (7), the growth rate is

$$
\left(\frac{\omega_{g}}{\omega_{o}}\right)_{\max }=\frac{I_{b}}{I_{o}} \frac{\beta_{\mathrm{ave}}}{\gamma} \frac{R}{2 b^{2}}
$$

The worst case is at injection ( $2 \mathrm{TeV}$ energy or $\gamma=2.13 \times 10^{3}$ ), and for the other parameters we take $2 \pi R=87 \mathrm{~km}, \beta_{\mathrm{ave}}=220 \mathrm{~m}, I_{b}=70 \mathrm{~mA}$ and a tube diameter $2 b=33 \mathrm{~mm}$. (For protons, $I_{o}=3.13 \times$ $10^{7} \mathrm{~A}$.) The normalized growth rate is

$$
\left(\frac{\omega_{g}}{\omega_{o}}\right)_{\max }=5.9 \times 10^{-3}
$$

with these parameters. This corresponds to an instability $e$-folding timescale $\left(\tau_{g}=1 / \omega_{g}\right)$ of 27 revolution periods $\left(\tau_{o}=2 \pi / \omega_{o}\right)$.

In the regime where the thickness of the conducting layer is less than a skin depth, but $\omega \tau_{m} \gg>1$, the growth rate is reduced from the maximum value by a factor of $2 / \omega \tau_{m}$ :

$$
\left(\frac{\omega_{g}}{\omega_{o}}\right)=\frac{I_{b}}{I_{o}} \frac{\beta_{\mathrm{ave}}}{\gamma} \frac{R}{b^{2} \omega \tau_{m}} .
$$

For example, increasing the $e$-folding time to 100 revolutions requires $\omega \tau_{m} \simeq 7.4$. The lowest allowed frequency is the fractional tune $n-v \equiv \delta v$ times the revolution frequency $\omega_{o}$, and from the definition of $\tau_{m}$ (Eq. (38)), the $\omega \tau_{m}=7.4$ condition requires

$$
\sigma \Delta \geqq 3.3 \times 10^{4} / \delta v
$$

For example, with copper and an RRR of $30\left(\sigma \simeq 2 \times 10^{9} \mathrm{mhos} / \mathrm{m}\right), \delta v=0.2$, the thickness must be larger than $82 \mu \mathrm{m}$. The skin depth in this example is $0.42 \mathrm{~mm}$, so our approximations are well satisfied with these layer thicknesses for the lowest frequency mode. For completeness, we give the general expression for the criteria on $\sigma \Delta$ for a desired instability growth per revolution $\left(\tau_{g} / \tau_{o}\right)$ :

$$
\sigma \Delta=\left(\frac{\tau_{g}}{\tau_{o}}\right)\left(\frac{1}{\delta v}\right) \frac{4 \pi R^{2} \beta_{a v e} I_{b}}{Z_{o} b^{3} \gamma I_{o}}
$$

(in the regime $\omega \tau_{m}>1$ ). 
It is interesting to note that the instability $e$-folding per revolution scales as $\mathrm{R}^{2}$; the SSC has relatively modest average currents, but the resistive mode is still significant because of this scaling (and the relatively small bore size that enters in the $1 / b^{3}$ factor in the growth rate).

The condition for stabilization of the resistive mode by a tune spread was derived in Section 2.0. At injection the space-charge tune spread is of order $10^{-3}$. This tune spread is not sufficient to stabilize the lowest frequency mode with the "baseline" parameters given above; however, the higher frequency modes could be stabilized. The criteria for stabilization, Eq. (14), can be written as

$$
\Delta v_{s} \geq \pi\left(\omega_{g} / \omega_{o}\right)
$$

or

$$
\geq \frac{\tau_{0}}{2 \tau_{g}}
$$

where $\omega_{g}\left(\tau_{g}\right)$ are the growth rates (times) in the absence of a tune spread calculated in Section 3.0. The lowest frequency mode has $\tau_{g} \sim 100 \tau_{o}$ with $\delta v=0.2$ and a copper layer of order $100 \mu \mathrm{m}$ (and a RRR of 30 ); the next higher mode has a growth rate 6 times smaller, which should satisfy the marginal stability condition. We also note that the space-charge tune spread scales with the individual bunch intensity, while the instability growth rate scales with average current. During the collider fill, or with operation at a reduced number of bunches, the lowest frequency mode can also be stabilized.

At full energy $(20 \mathrm{TeV})$, the instability growth rate is reduced by one order of magnitude from its injection value (the $1 / \gamma$ factor in Eq. (7)). When the beams are brought into collision, the beam-beam tune spread with the SSC baseline parameters is sufficient to stabilize all of the transverse resistive instability modes. The instability feedback stabilization systems may not be needed when operating with colliding beams. 


\section{REFERENCES}

1. J. D. Jackson, SSC Central Design Group, Surface Impedance of a Two-Layer Beam Tube and Resistive-Wall Contribution to Machine Impedances, January 1986.

2. SSC Conceptual Design Report, March 1986.

3. The spread in betatron frequencies from nonlinear space charge forces can be modeled in this fashion. For a comparison with particle simulation codes in the case of a selfpinched beam, see (for example) E. P. Lee, Phys. Fluids 21, 1327 (1978) and M. Lampe, W. M. Sharp, R. F. Hubbard, E. P. Lee, and R. J. Briggs, Phys. Fluids 27, 2921 (1984). 


\section{APPENDIX}

In this appendix, the general solution for the electric and magnetic fields is obtained without any constraints on the wall conductivity. The specific example we consider is a "thin" conducting cylinder $(\Delta<<\delta)$ of radius $b$, equivalent to a conducting sheet of "surface" conductivity $\sigma_{s}=\sigma \Delta$.

In part, the motivation for considering this system was the suggestion that a synchrotron radiation intercept of very low conductivity might be constructed that would be "transparent" to the low-frequency resistive instability fields. This would allow for high conductivity layers at larger radii to terminate the fields, thereby lowering the interaction impedance through its $1 / b^{3}$ dependence. The general field solution derived here shows that the charge relaxation timescale would have to be very long for this scheme to work, and this is difficult to achieve in practice.

The transverse fields inside the cylinder $(r \leq b)$ are given by Eqs. (15)-(18), assuming long wavelengths and low frequencies, so that

$$
\begin{aligned}
& k b \ll 1 \\
& \frac{\omega b}{c} \ll 1 .
\end{aligned}
$$

The transverse magnetic field outside the cylinder is given by Eq. (31), while the transverse electric field is of the form

$$
\bar{E}=E_{0} \frac{b^{2}}{r^{2}}\left[\sin \theta \bar{a}_{r}-\cos \theta \bar{a}_{\theta}\right]
$$

Using Eq. (20), the general solution for the axial electric field inside the cylinder $(r \leq b)$ is

$$
E_{z}=j k\left(\frac{Z_{o} I_{b} Y_{b}}{2 \pi r}-E_{w} r\right) \sin \theta-j \omega \mu_{o}\left(\frac{I_{b} Y_{b}}{2 \pi r}+H_{w} r\right) \sin \theta,
$$

while outside the cylinder $(r \geq b)$ it is

$$
E_{z}=j k E_{o} \frac{b^{2}}{r} \sin \theta-j \omega \mu_{o} H_{o} \frac{b^{2}}{r} \sin \theta
$$

In a similar fashion, using

$$
\frac{1}{r} \frac{\partial H_{z}}{\partial \theta}-\frac{\partial H_{\theta}}{\partial z}=-j \omega \varepsilon_{o} E_{r}
$$


we have the axial magnetic field in $r \leq b$ :

$$
H_{z}=j k\left(\frac{I_{b} Y_{b}}{2 \pi r}-H_{w} r\right) \cos \theta-j \omega \varepsilon_{o}\left(\frac{Z_{o} I_{b} Y_{b}}{2 \pi r}+E_{w} r\right) \cos \theta,
$$

and

$$
H_{z}=j k H_{o} \frac{b^{2}}{r} \cos \theta-j \omega \varepsilon_{o} E_{o} \frac{b^{2}}{r} \cos \theta
$$

in $r \geq b$.

The continuity conditions on $E_{z}$ and $H_{\theta}$ are given in Eqs. (33) and (34), while the remaining conditions are

$$
\begin{gathered}
E_{\theta}\left(b^{+}\right)=E_{\theta}\left(b^{-}\right) \\
H_{z}\left(b^{+}\right)-H_{z}\left(b^{-}\right)=-\sigma \Delta E_{\theta}(b) .
\end{gathered}
$$

Using these continuity conditions, we can solve for the transverse field amplitudes that enter into the collective transverse force on the beam $\left(E_{w}, H_{w}\right)$. Eliminating $E_{o}$ and $H_{o}$, we have

$$
\begin{gathered}
H_{w}\left(1+j \omega \tau_{m}\right)+j \omega \tau_{m} \frac{I_{b} Y_{b}}{2 \pi b^{2}}=-\omega \varepsilon_{o} k b^{2} E_{w}-k^{2} b^{2} H_{w} \\
E_{w}\left(1+j \omega \tau_{E}\right)-\frac{Z_{o} I_{b} Y_{b}}{2 \pi b^{2}}=-j \frac{2 k b}{\sigma \Delta} H_{w}
\end{gathered}
$$

where the magnetic time constant $\tau_{m}$ is given by Eq. (38), and the electric (charge relaxation) time constant is defined by

$$
\tau_{E}=\frac{2 \varepsilon_{o} b}{\sigma \Delta}
$$

Solving Eqs. (60) and (61) for $E_{w}$ and $H_{w}$, we have

$$
\begin{aligned}
& H_{w}\left(1+j \omega \tau_{m}\right)+j \omega \tau_{m} \frac{I_{b} Y_{b}}{2 \pi b^{2}}=-\frac{k^{2} b^{2}}{1+j \omega \tau_{E}} H_{w}-\frac{\frac{\omega b}{c} k b}{1+j \omega \tau_{E}} \frac{I_{b} Y_{b}}{2 \pi b^{2}} \\
& E_{w}\left(1+j \omega \tau_{E}\right)-\frac{Z_{o} I_{b} Y_{b}}{2 \pi b^{2}}=\frac{k^{2} b^{2}}{1+j \omega \tau_{m}}\left[\frac{j \omega \tau_{E}}{1+j \omega \tau_{E}}-\frac{\omega}{k c}\right] \frac{Z_{o} I_{b} Y_{b}}{2 \pi b^{2}}
\end{aligned}
$$


Given the low-frequency, long-wavelength approximations already made (Eqs. (50) and (51)), the righthand side of these equations should be insignificant compared to the left-hand terms. In addition, we note from the definitions of $\tau_{m}$ and $\tau_{E}$ that

$$
\tau_{m} \tau_{E}=b^{2} / c^{2}
$$

Only one of the $\omega \tau_{m}, \omega \tau_{E}$ terms can be significant compared to unity, since

$$
\left(\omega \tau_{m}\right)\left(\omega \tau_{E}\right)=\omega^{2} b^{2} / c^{2}<<1
$$

We therefore have two regimes, "magnetic" or "electric", depending on the value of the surface conductivity $\sigma \Delta$. From the definitions of $\tau_{m}$ and $\tau_{E}$, we note that

$$
\frac{\tau_{m}}{\tau_{E}}=\left(\frac{Z_{o} \sigma \Delta}{2}\right)^{2}
$$

(a) Magnetic regime:

When $\sigma \Delta \gg 2 / Z_{o}$, as is usually the case, $\tau_{E}<<b / c$, and the approximate solution for the fields is

$$
E_{w}=\frac{Z_{o} I_{b} Y_{b}}{2 \pi b^{2}}
$$

and

$$
H_{w}=-\frac{j \omega \tau_{m}}{1+j \omega \tau_{m}} \frac{I_{b} Y_{b}}{2 \pi b^{2}}
$$

resulting in the transverse interaction impedance given by Eq. (37).

(b) Electric regime:

If $\sigma \Delta \ll 2 / Z_{O}, \tau_{m}$ is much less than $b / c$, and the electric charge relaxation timescale dominates. The transverse fields are

$$
E_{w}=\frac{1}{\left(1+j \omega \tau_{E}\right)} \frac{Z_{o} I_{b} Y_{b}}{2 \pi b^{2}}
$$

and the magnetic field from wall image currents is negligible, since

$$
\left|H_{w}\right| \ll<\left(\frac{\omega b}{c}\right) \frac{I_{b} Y_{b}}{2 \pi b^{2}}
$$

The interaction impedance is the same as Eq. (37), with $\tau_{E}$ replacing $\tau_{m}$.

With metallic conductors, of course, the electric regime is never encountered, since the thickness would need to be smaller than atomic dimensions! 
